Bull. Mater. Sci., Vol. 21, No. 4, August 1998, pp. 335-339. (C Indian Academy of Sciences.

\title{
Sliding wear studies in glass-epoxy system through scanning microscopic observations
}

\author{
P SAMPATHKUMARAN, S SEETHARAMU, A MURALI, R K KUMAR and KISHORE* \\ Materials Technology Division, Central Power Research Institute, Bangalore 560 094, India \\ *Department of Metallurgy, Indian Institute of Science, Bangalore 560012 , India
}

MS received 19 December 1997

\begin{abstract}
The work reports the wear occurring in a glass-epoxy composite system as a function of sliding distance for a constant sliding velocity and applied load. It is seen that the weight loss increases with increasing distance, but the gradient maintained all through is not the same. An attempt has therefore been made to correlate the weight loss with the scanning microscopic observations on the worn surfaces. It is found that in the early part, the wear of the resin/mat layer contributes to the phenomenon. The process of breakage of fibre into fragments and the resin debris formation accounts for the wear at a much later stage. The phenomenon occurring in between these two stages, is attributed to some of the broken fibres getting lodged in the matrix and agglomeration of the debris formed from the matrix material. Other features of interest are highlighted and discussed in this report.
\end{abstract}

Keywords. Glass-epoxy; pin-on-disc; sliding wear; SEM observations.

\section{Introduction}

The use of polymers and their composites are on the increase for improved performance in many gear, bearing and lining applications. With such newer materials, complex parts could be made with ease and at the same time economically viable. The additional advantages that are gained in polymeric systems are the ability to absorb shock/vibration, and to operate with less power and noise with very little maintenance. The use of fillers, reinforcements, and lubricants in the matrix resin, gives rise to many combinations that provide increasing load withstanding capability, reduced coefficients of friction, improved wear resistance and thermal properties, higher mechanical strength etc. Internally lubricated composites with thermal stability and increased resistance to wear is an area wherein research is being carried out (ASM 1992).

Generally, the frictional properties of polymers are different from that of metals. In contrast to metals, reinforced epoxy resins deform at higher loads. The friction at the mating surfaces is characterized by adhesion and deformation. As a result, the coefficient of friction decreases as the load is increased. Also the frictional force is recorded to be proportional to speed (Friedric 1986). The wear of polymeric composites with different reinforcements is the topic of investigation in recent times (Vishwanath et al 1991, 1992; Sinha and Biswas 1992, 1995).

*Author for correspondence
The wear rate of a given material is a measure of the weight changes in the material with respect to time. The material removal from the sample in contact with a hard surface is characterized by several mechanisms, the primary one being adhesive wear, wherein fine particles of polymer gets removed from the surface. On the other hand, the presence of either the fused polymer or the grooves at the interface is interpreted to indicate that the materials are wearing out by abrasion instead of adhesion (ASM 1992).

One of the important composites that is widely used is the well known glass fibre reinforced polymer (GFRP) material. The rigidity and strength of polymeric materials are less by 25 times when compared with that of glass. However, as regards the thermal expansion of glass, it is 25 times smaller than that of polymer. Generally, the diameter of these glass fibres are in the range 8-12 $\mu \mathrm{m}$. They are used either in the form of chopped fibres or as filaments $0.1-5 \mathrm{~mm}$ long, for reinforcing thermoplastics (Lothar Engel et al).

The most commonly used resins are the epoxy resins. They possess useful mechanical properties and are versatile. Further they wet many substrate materials, absorb less moisture and above all can be processed with considerable degree of ease. Among the other advantages worth mentioning are that they have excellent chemical resistance coupled with good electrical properties (Lee and Nevilee 1967).

The epoxy resin can be processed into a variety of useful products like protective coatings, adhesives, struc- 


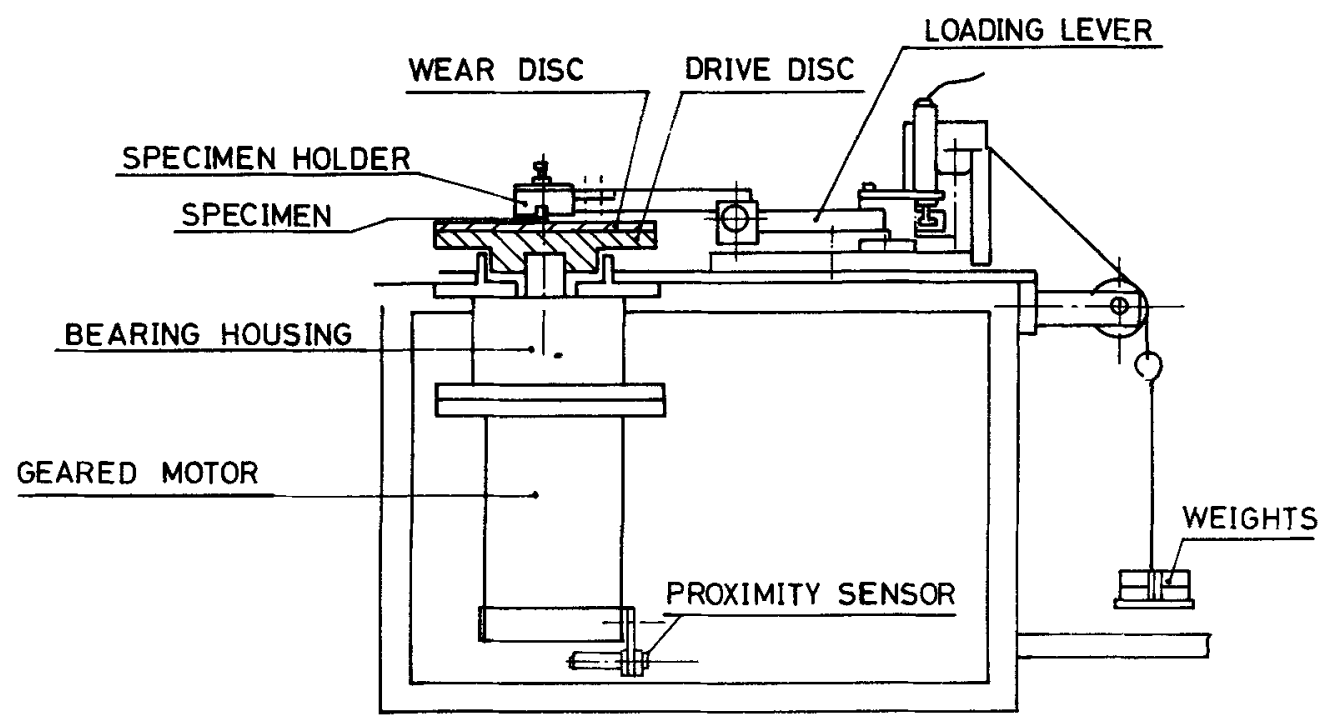

Figure 1. A schematic of pin-on-disc set up showing the different parts of the sliding wear system.

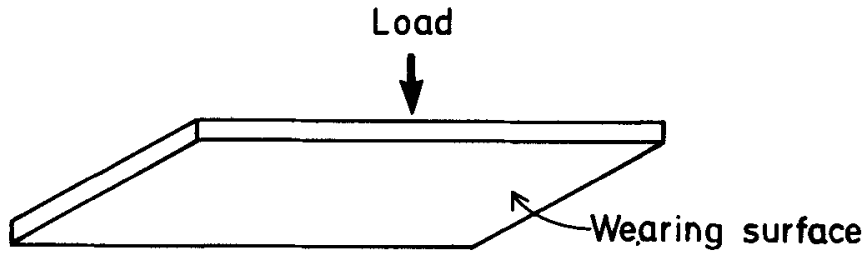

Figure 2. A schematic diagram showing the flat specimen with the load applied on the top face and the bottom face subjected to wear by contact with the rotating disc.

tural components with appropriate curing agents (Lee and Nevilee 1967). They usually withstand service temperature up to $160^{\circ} \mathrm{C}$, whereas multifunctional resins have been reported to operate even at $250^{\circ} \mathrm{C}$ without deterioration in properties (Purslow 1984).

In this work, therefore, epoxy resins reinforced with bidirectional glass fabrics are made into laminates and the tribological property involving weight changes due to dry sliding against a steel disc is characterized by observations made in the scanning electron microscope (SEM). This approach was adopted to elucidate the mechanism of wear in the composite.

\section{Materials and methods}

The glass-epoxy material used in this investigation was made from the hand lay-up technique using a compression moulding technique. The procedure consisted of placing the glass fibres with epoxy compatible finish on a substrate material which had release coat on it. Weighed quantities of epoxy resin mix was taken and smeared over this glass fabric which was bidirectional in nature.

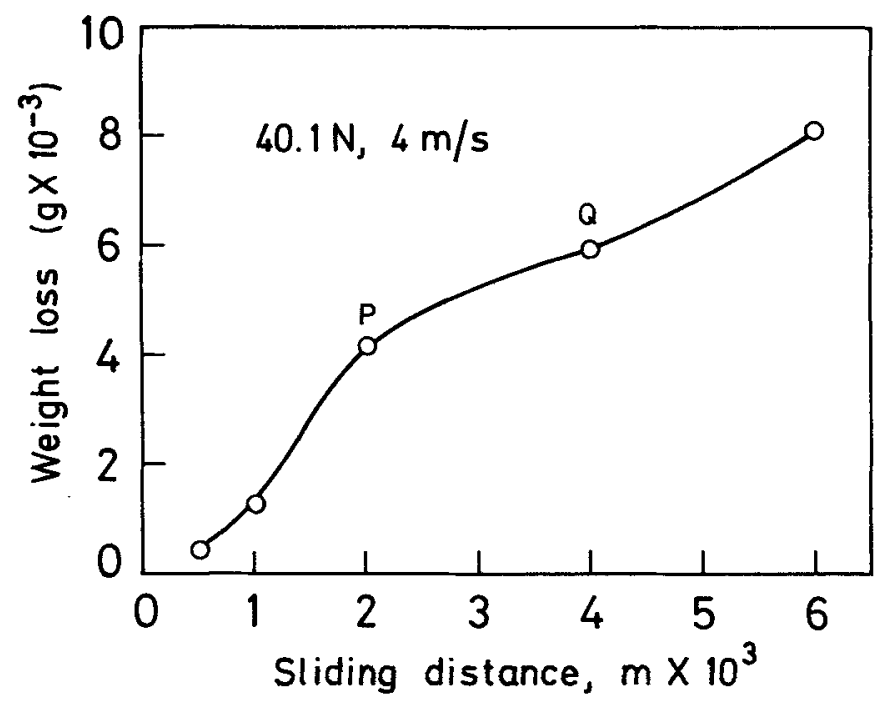

Figure 3. A plot showing the weight loss vs sliding distance.

On this, another layer of the glass fabric was laid and the process continued to yield laminates of the required resin reinforcement ratio.

The whole lay up covered with a mat finished fabric over it was placed on a steel plate with the necessary release coat applied on it. The entire lay up was pressed in a press to yield laminates of about $3 \mathrm{~mm}$ thickness. The composite system was cured at ambient conditions for a period of $24 \mathrm{~h}$. The laminate thus made was cut using a diamond tipped cutter in order to have a smooth finish. Samples of size $6 \times 6 \mathrm{~mm}$, for the wear test, were later cut from the cured laminate.

The test samples were then glued to pins of size $6 \mathrm{~mm}$ dia. $\times 25 \mathrm{~mm}$ length using suitable adhesive and their 
initial weights recorded using a high precision digital electronic balance after thorough cleaning. The hardened alloy steel material (62 HRC) having surface roughness of $\mathrm{Ra}=10 \mu \mathrm{m}$ was used as the disc.

After fixing both the disc and the sample pin in their respective positions, the normal load to the pin was applied through a pivoted loading lever with a string and pan assembly as elaborated in figure 1 . The mounting arrangement of the sample consisted of making the flat face of the lay up come in contact with the rotating hardened steel disc (figure 2). After the preset time is reached, the test was stopped using the timer mechanism provided in the machine. The weight of the sample after the completion of the test was measured.

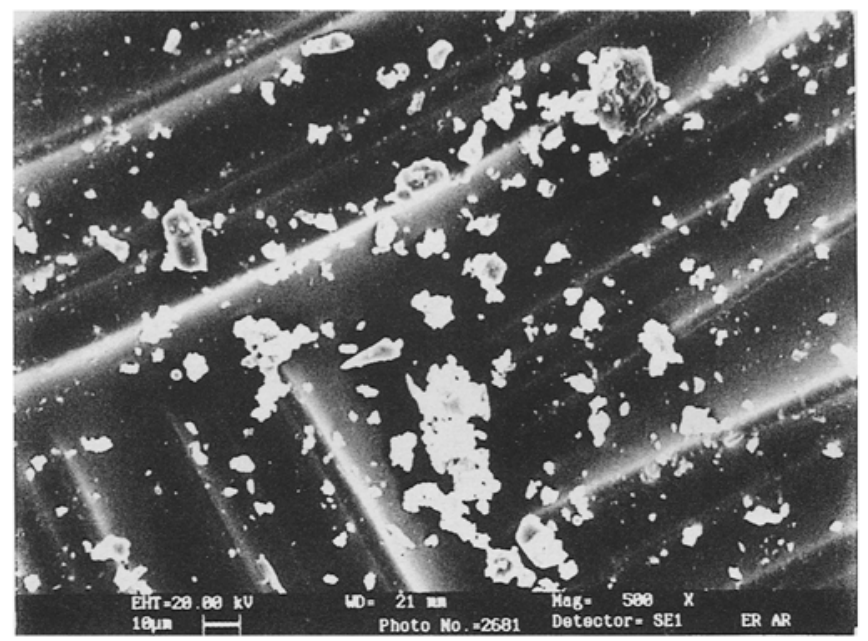

Figure 4. Scanning electron microscopic picture of the as-received sample showing mainly the regions of fibres and matrix material.

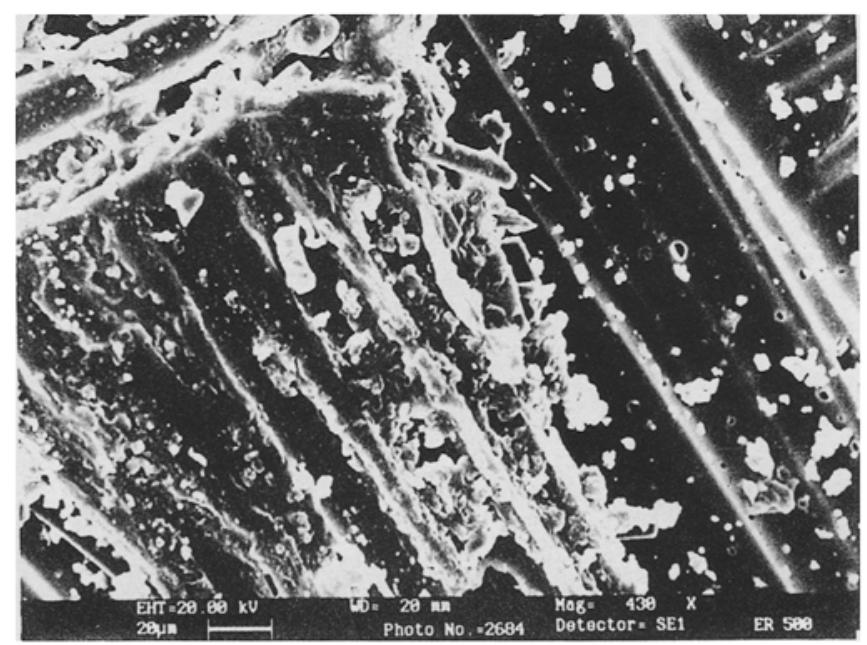

Figure 5. SEM view of a sample subjected to wear for a distance of $500 \mathrm{~m}$.
The samples were subjected to normal load of $40 \mathrm{~N}$. The disc was rotated continuously till select distances in the range $500-6000 \mathrm{~m}$ were reached and the weight loss changes recorded. In a select few samples, after the required wearing distances were reached, scanning electron microscope (SEM) examinations were conducted. All the experiments were carried out at sliding speed of $4 \mathrm{~m} / \mathrm{s}$. In one specific case only, the sample was subjected to a higher load of $60 \mathrm{~N}$ and made to slide for a larger distance of $6000 \mathrm{~m}$ at a higher sliding speed of $5 \mathrm{~m} / \mathrm{s}$, with a view to accelerate the wearing process and examine the picture that would emerge. In this specific case, only SEM observations were carried out as was the prime purpose of this reporting. A minimum of four test runs were carried out for each set of test conditions for recording the weight changes. The samples being non-conducting, they were sputter coated with a layer of gold before the SEM examination. The features of interest were noted and the regions of importance photographed.

\section{Results and discussion}

Figure 3 gives the weight loss due to wear against sliding distance for a constant load of $40 \mathrm{~N}$ and sliding velocity of $4 \mathrm{~m} / \mathrm{s}$. It is obvious from the graph that the wear is initially high and at a distance of $2000 \mathrm{~m}$, the wear rate comes down considerably indicating a change in the wear process. With a view to get a better comparative picture, the sample without subjecting to wear (i.e. the as received material) was viewed in SEM and the features were recorded (figure 4).

Figures 5-8 depict the SEM features of worn out samples taken out at different sliding distances. Thus figure 5 illustrates the features at $500 \mathrm{~m}$. It may be

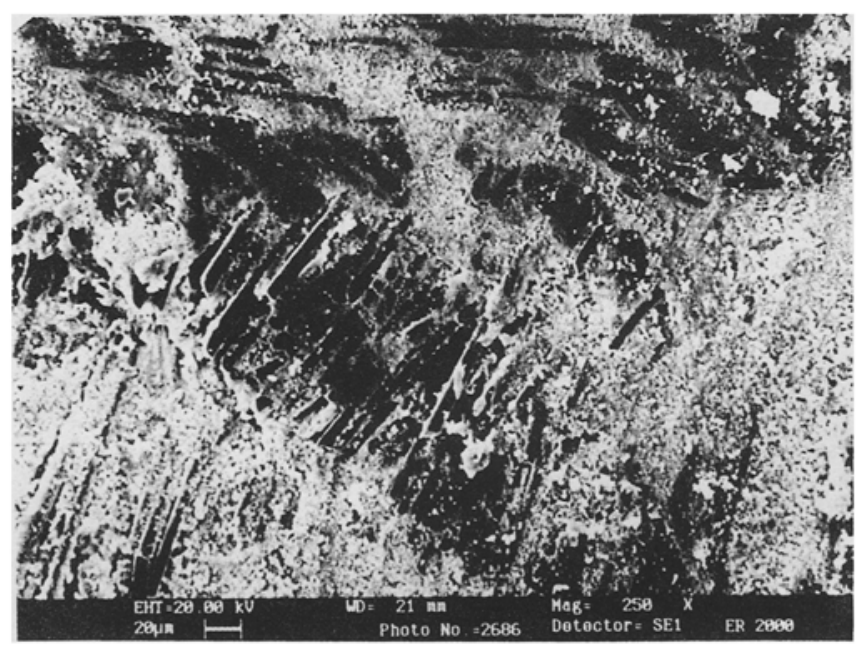

Figure 6. A SEM photograph showing debris formation and glass fibre rich regions in a sample run for a distance of $2000 \mathrm{~m}$. 
observed from this figure that the resin is still adhering to the glass fibre like in the as received sample (figure 4). However, the adhering regions seen in the as received sample yield place to debris formation due to wear.
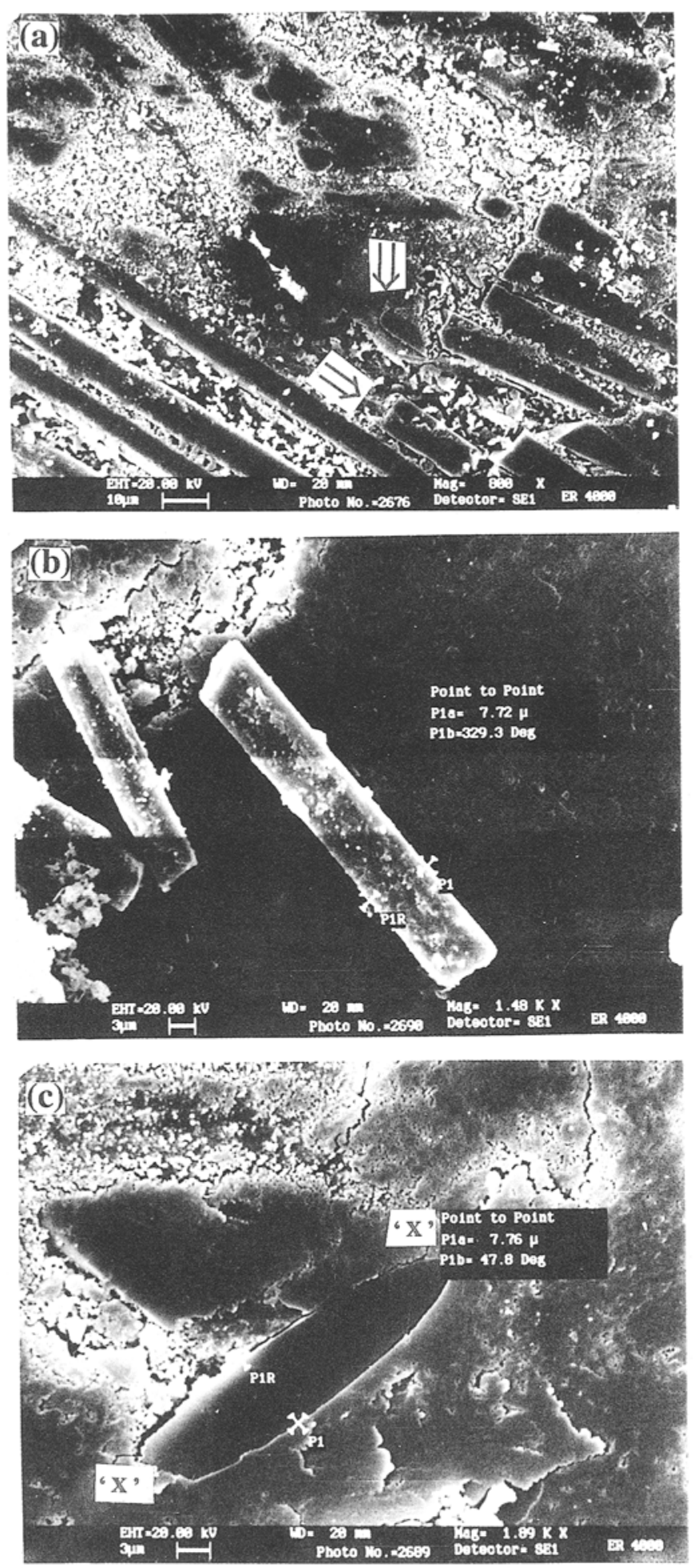

Figure 7. The features seen in sample examined after a $40(0)$ $\mathrm{m}$, sliding depicting (a) debris formation and broken fibres; (b) two broken and dislodged fibres and (c) a fragmented fibre lodged in the matrix rich regions.
When dry sliding was allowed to occur in this manner, the first noticeable difference was seen in the case of samples whose features are detailed in figure 6 . In this condition the appearance of small debris in large quantities throughout the sample under view can be seen. Also noticed are the glass fibres which are protruding because of the removal of resinous material from the surface. The glass fibres of considerable length in a few numbers can be noticed in this condition i.e. $2000 \mathrm{~m}$ sliding distance (figure 6).

When the test was continued further, at a sliding distance $4000 \mathrm{~m}$, broken and fragmented glass fibres at regions marked by arrows can be seen (figure 7a). Also of interest to note is that the resinous material is well spread out. The debris that were formed (figure 7a), tended to agglomerate by a kind of cold welding process. Also, broken fibres of about $7.7 \mu \mathrm{m}$ diameter can be seen (figure 7b). The process of wear of the matrix resin must have helped in dislodging such glass fibres. Also within the coalesced debris region, features resembling intergranular cracks in metals (figure $7 \mathrm{c}$ ) can be seen. The glass fibre not only is fragmented, but also forms an inclined fracture near its edge (indicated by ' $X$ '; figure 7c). When the test was carried out for 6000 $\mathrm{m}$ and run at a higher load $(60 \mathrm{~N})$ and higher speed $(5 \mathrm{~m} / \mathrm{s})$, extensive debris formation was seen along with broken fibres (figure 8 ).

The above photographs indicate that the wear initially is controlled by the removal of mat layer, used to encompass the laminate, and the resin. This resin layer removal leading to debris formation continues even after $2000 \mathrm{~m}$ of sliding by the test sample. Subsequent to this wearing process, the sub-surface involving the glass fabric contributes to the observed loss in material. The hard broken fibres getting lodged in the resinous material

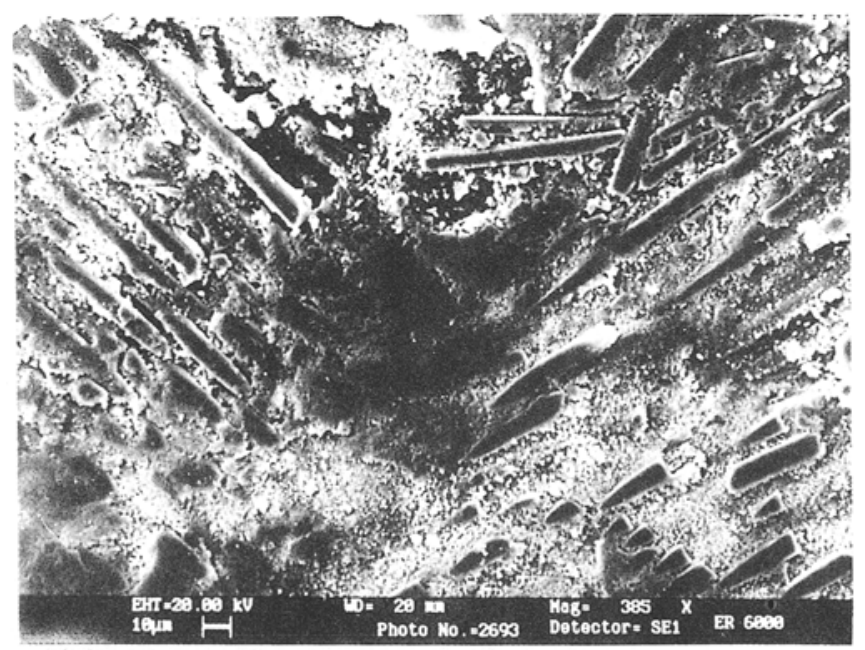

Figure 8. A photograph showing a larger proportion of fragmented fibres in a sample subjected to wear for a longer sliding distance of $6000 \mathrm{~m}$, and a higher load of $60 \mathrm{~N}$. 


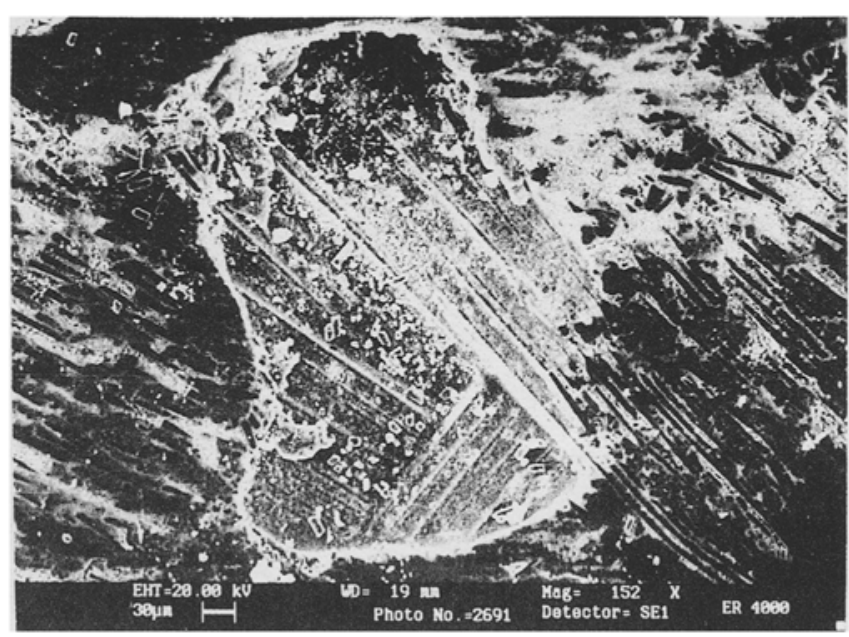

Figure 9. Formation of craters revealing the fabric arrangement in the lower plies.

as well as the agglomeration of the loose debris contribute to a decrease in the kinetics of wear leading to a lowering of the gradient seen in the graph (figure 3 , in between the regions marked $P$ and $Q$ ). However, after about $4000 \mathrm{~m}$ sliding, an upward trend in the gradient of the wear loss is seen (figure 3). This is attributed to the extensive loss of material due to fragmentation of glass fibres and their detachment from the test material due to the non availability of a medium to hold them. Also in the case of $4000 \mathrm{~m}$ run sample, the central portion shows a crater like feature indicating that a big chunk of material is affected by the wear process for longer duration run (figure 9). Due to this lift-off, the underneath layer of glass fibre with epoxy is very well exposed in the lower half of the crater. In the upper half of the crater, a depression bounded by an arc like resinous region is seen. Also of significance to note is that the fragmented fibres are inclined in the direction of the movement of sample thus stressing the role played by the tangential stresses in orienting the fragmented fibres (figure 10).

\section{Conclusion}

The present experimental approach points out the differences in the role played by the resin and the glass fabric medium towards the sliding wear situation in the GFRP type composites. While the initial wear process is controlled by the loss of resin leading to debris formation, the breaking up of long continuous fibres into

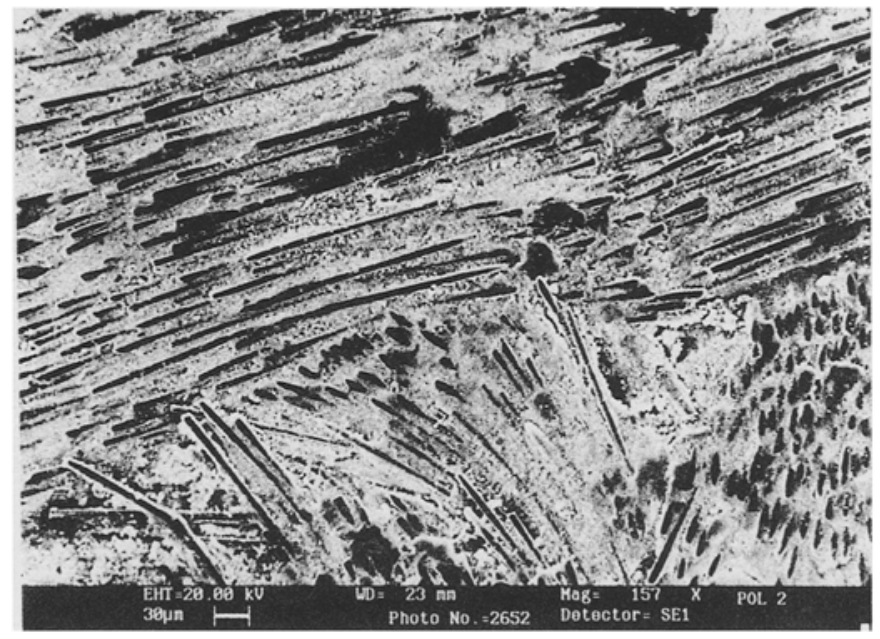

Figure 10. A region of the sample exhibiting the bending of the glass fibres.

small pieces and the loss of some of these fragments when they are not held by the matrix contributes to wear loss at a later stage.

\section{Acknowledgements}

The authors would like to acknowledge the assistance of Shri D Mollaiah for the help rendered and Prof. D H Sastry, Department of Metallurgy, Indian Institute of Science, Bangalore for the keen interest shown in the work.

\section{References}

ASM Handbook 1992 (Materials Park, USA; ASM International) Vol. 18

Friedric K 1986 Friction and wear of composites, in Workshop on centre for composite materials (USA: University of Delaware)

Lee $H$ and Nevilee K 1967 Handbook of epoxy resins (New York: Mc Graw Hill) p. I-5

Lothar Engel, Hermann Klingele, Gottfried W E and Helmut Schaper An atlas of polymer damage (Vienna: Wolfe Science Books and Carl Hanser, Verlag) Translated by M S Welling Purslow D 1984 Composites 1543

Sinha S K and Biswas S K 1992 J. Mater. Sci. 273085

Sinha S K and Biswas S K 1995 J. Mater. Sci. 302430

Vishwanath B, Verma A P and Kameswar.. Rao C V S 1991 Wear 145315

Vishwanath B, Verma A P and Kameswara Rao C V S 1992 Comp. Sci. \& Technol. 4477 